\title{
Migración del campo a la ciudad: experiencias de mujeres del Bañado Sur. Asunción 2019
}

\section{Migration from the countryside to the city: experiences of women from the South Banado. Asuncion 2019}

\author{
Belinda García ${ }^{\circledR}$
}

\begin{abstract}
Resumen
La investigación buscó describir la experiencia migratoria del campo a la ciudad de mujeres del Bañado Sur de Asunción en el 2019, a través del diseño cualitativo, descriptivo, observacional; y a través de la mirada de mujeres migrantes con elementos de la realidad empírica. Desde la perspectiva crítica se logra caracterizar el fenómeno migratorio con relación al problema de la tierra en Paraguay. Ésta entendida como relación social quebrada mediante estos desplazamientos forzados que finalmente obedecen a cambios producidos al interior de la estructura económica de producción.

Se ponen entonces en evidencia las características sociodemográficas de las mujeres migrantes que entrelazan concomitantemente desigualdades sociales en el medio urbano como en el rural, dirigiéndolas hacia la precarización laboral en ambos medios.

Estas mujeres habían realizado un tránsito previo a otras áreas geográficas y no directamente desde sus comunidades de origen hasta el Bañado Sur; es decir, se dio un proceso de migración rural-rural y rural-urbana.

Fueron identificados seis momentos de tránsito: 1) la actualidad, en el área urbana como último asentamiento 2) antes de la migración, donde se desarrollaba la vida en el área rural antes del arribo a las ciudades 3) el día de la migración, que constituye un evento angular en la vida de las mujeres 4) el momento migratorio de tránsito de la comunidad de origen, que posibilitó observar la movilidad en su contexto social 5) el momento dellegada, que configura las condiciones en que las ciudades reciben a las mujeres, el primer reasentamiento; y finalmente 6) la transición en la ciudad y lo que conlleva iniciar la vida con otros parámetros, códigos y lenguajes, que no deja de ser un proceso inacabado, ya que es multidimensional.

Palabras clave: migración, género, bañados, área rural, área urbana.
\end{abstract}

1 Universidad Nacional de Asunción, Facultad de Ciencias Sociales. San Lorenzo, Paraguay.

Correspondencia:

beligarciapy@gmail.com

Recibido:

4 de setiembre de 2021

Aceptado:

28 de octubre de 2021

Doi:

https://doi.org/10.54549/ky.6e.2021.62

(c)
Artículo publicado en acceso
abierto bajo la Licencia Creative
Commons.
Cita:
García, B. (2021). Migración
del campo a la ciudad:
experiencias de mujeres del
bañado sur. Asunción 2019.
Kera Yvoty: reflexiones sobre
la cuestión social, 6(número
especial), 62-76. https://doi.
org/10.54549/ky.6e.2021.62

\begin{abstract}
The research sought to describe the migratory experience from the countryside to the city of women of Bañado Sur de Asunción in 2019, through the qualitative, descriptive, observational design; and through the look of migrant women with elements of empirical reality. From the critical perspective is able to characterize the migratory
\end{abstract}


phenomenon with relation to the land problem in Paraguay. It is understood as social relationship broken through these forced displacements that finally obey to changes produced within the economic structure of production.

The sociodemographic characteristics are then highlighted of migrant women who concomitantly intertwine social inequalities in urban and rural areas, directing them towards job insecurity in both means. These women had made a previous transit to other geographical areas and not directly from their communities of origin to the Bañado Sur; that is, there was a process of rural-rural migration and rural-urban.

Six moments of transit were identified: 1) the present, in the urban area as the last settlement 2) before migration, where life developed in the rural area before arriving at the cities 3 ) the day of migration, which constitutes an angular event in the lives of women 4) the migratory moment of transit of the community of origin, which made it possible to observe mobility in its social context 5) the moment of arrival, which configures the conditions in which cities receive women, the first resettlement; and finally 6 ) the transition in the city and what it entails to start the life with other parameters, codes and languages, which is still a unfinished process, as it is multidimensional.

Keywords: migration, gender, wetlands, rural area, urban area.

\section{Introducción}

El presente trabajo está orientado en el debate de la migración interna, mediante una estructura enmarcada en la problematización del fenómeno migratorio, vinculado a categorías específicas desde la perspectiva de género por lo que se pretende abordar el fenómeno desde un enfoque global. Además, la perspectiva histórico-crítica posibilita hacer visibles las desigualdades en lo económicoy en lo social; logrando así, vincular elementos teóricos con las vivencias en las comunidades donde habitan estas mujeres (Salazar Cruz, 200o). Sin embargo, cabe aclarar que no existe una conceptualización acabada de estos fenómenos de migración interna ya que son procesos multidimensionales.

La producción del conocimiento está en constante movimiento, los paradigmas epistemológicos del cómo se establecen las formas de entender los fenómenos migratorios están llenos de compromisos con ideas de orden social (Carpinetti, 2017) pero también están enmarcadas en las tensiones que genera el sistema desigual del modelo de producción capitalista. Así, se elaboraron interpretaciones del proceso social en general con una importante atomización.

Los estudios de la migración se han desarrollado desde varias perspectivas, en su mayoría se han presentado las causas, no en igual medida los efectos (Gómez Walteros, 2010). En el área específica del trabajo social como campo disciplinar hay escasos antecedentes del estudio del fenómeno a nivel nacional. Es innegable la existencia de estos desplazamientos forzados que generan nuevas dinámicas en los territorios en distintas épocas de la historia (Ocampo, 2015).

El desplazamiento migratorio es fruto de una violencia que se encuentra inscripta estructuralmente en la sociedad (Osorio, 1993) pero sin embargo existen autores que afirmaron que la migración como fenómeno estaba estimulada por consideraciones individuales racionales así las causas de la migración fueron el objeto de estudio dentro del paradigma positivista, y que en general se presentaron como la suma de decisiones racionales de sectores económicos con diversos grados de desarrollo (Carpinetti, 2017).

Existen diversos enfoques teóricos en cuanto al abordaje conceptual de lo que significa migrar (Riquelme \& Vera, 2015) pero estos se basan en dos teorías principales: Una, la de modernización, que presenta al fenómeno como un proceso lineal marcado por la evolución social, o sea que se transita de lo arcaico 
a lo moderno; y otra desde la perspectiva histórica estructural, que exige una mirada dentro de la dinámica del capitalismo con sus relaciones contradictoras y lo ve como un resultado de los cambios que operan al interior de la estructura económica de producción. Si hacemos referencia a la transición desde un espacio rural a un espacio urbano podemos decir que la migración es una transición transformadora que no quiere decir progreso (Salazar Cruz, 2000).

Es en este debate en el cual se pretende enfocar el fenómeno $y$ en términos específicos la aproximación al estudio de la migración interna desde el campo a la ciudad, que comúnmente suele denominarse también como desde lo rural a lo urbano, varios estudios que tienen como eje los desplazamientos internos hacia los centros urbanos mencionan que las mujeres son claves para dichos procesos (Antonelli, 2016; Ocampo, 2015; Riquelme \& Vera, 2015).

La migración vista desde una perspectiva de género va evidenciando las desigualdades (Antonelli, 2016) que padecen las mujeres en la cultura patriarcal entrelazada con las contradicciones del capitalismo. Esto permite también reconocer que no puede asumirse que la migración implique necesariamente el empoderamiento de las mujeres.

Para el estudio del fenómeno migratorio a nivel nacional es necesario tener en cuenta la principal problemática del Paraguay en su estructura social. El problema de la tierra es la génesis esencial de la desigualdad (Palau \& Heikel,2016) y por consiguiente se ubica al fenómeno migratorio como un producto de esta relación desigual en la tenencia de la tierra.

La problemática de la migración está estrechamente vinculada al problema de la tierra. Este proceso de movilidad está visto como forzado. En él están afectadas las comunidades campesinas e indígenas; donde habitan mujeres, niñas y personas adultas mayores, hombres y niños que finalmente fueron alcanzados por la modernización de la agricultura. Implicó también la alteración de la base social en la estructura productiva y generó campesinas y campesinos sin tierra; resultado de las imposiciones del capital transnacional (Palau \& Heikel, 2016).

Las mujeres en la construcción del mundo social han sido siempre vistas como el opuesto al otro, o sea a los hombres. Las mujeres han realizado las tareas del hogar y fueron el sostén estructural de las sociedades (Henault, 1973), pero este trabajo esencial fue relegado a un rol secundario mediante la jerarquización del trabajo social, la división sexual del trabajo y el denominado trabajo productivo que era otorgado a los hombres; obligándolas al cumplimento de un rol obligatorio ligada a la reproductividad y al género.

El sistema patriarcal elabora culturalmente representaciones respecto a los géneros, les establece roles que aseguran una configuración desigual desde lo simbólico hasta lo material. Ordena las relaciones sociales en jerarquías con un entramado de valores sobre el sistema binario que sobrepasa lo simbólico y se evidencia perfectamente en el ámbito de lo económico (Quiroga Diaz, 2009).

El capitalismo como sistema combinado con paradigmas patriarcales muestra claras contradicciones. En su desarrollo inserta las mujeres en el mercado, y si bien existe un mayor ingreso de mujeres al mercado mediante las oportunidades de empleabilidad que finalmente les permite mayores niveles de autonomía, es también cierto que la inserción de estas mujeres se corresponde a la mayor flexibilización laboral que a su vez implica el deterioro de las condiciones laborales y de reproducción de la vida (Quiroga Díaz, 2009).

En los procesos migratorios trasnacionales o internamente en los países, las mujeres son el epicentro de la movilidad, que se ha caracterizado como forzada. Este contexto migrante del campo a la ciudad (Ocampo, 2015) se sitúa dentro de un proceso de movilidad enmarcado en el dominio y control económico de grupos 
específicos en los territorios, que a su vez contribuyen a generar en las comunidades rupturas imposibles de describir con un primer abordaje puesto que implican secuelas, siendo la principal la pérdida del arraigo.

La crisis del capitalismo mundial y su impacto en América Latina, donde las economías fueron forzadas a la reconversión de grandes ramas que involucraban medidas de modernización y contemplaban inversión tecnológica y sociales (Marini, 1993), significó la afectación a diversos grupos sociales como también una mayor competencia entre grandes grupos económicos.

La incorporación de las mujeres a los mercados laborales mediante la inserción de América Latina al mercado internacional, lejos de significar una redistribución igualitaria de los ingresos reasigno a las mujeres el carácter de su clase. Esta reasignación de clase dio pie a la migración de mujeres desde las periferias para su ocupación en la actividad reproductiva y doméstica (Quiroga Díaz, 2009).

Cuando se mencionan a los asentamientos urbanos en América Latina puede verse que estos comparten características en lo territorial, ya sea en el escaso acceso a servicios públicos desde lo más básicos con son el agua, la luz y el gas, también se encuentran sin transporte, sin escuelas y sin puestos de salud. Mientras que en lo productivo tienen que ver con el aumento de la desocupación y la subocupación generando un desplazamiento constante para generar mayor productividad que da como consecuencia a su vez la progresiva consolidación del sector informal. Consecuencias al fin de la falta de políticas públicas, de organización territorial que tengan una multimirada hacia los aspectos que las comunidades requieran y en específico las mujeres. Así las sociedades han conformado el espacio urbano con amplias desigualdades de género (Falú, 2018).

Las migraciones registradas en este estudio datan desde el año 1971 hasta el último registro del año 2009.

\subsection{La migración como resultado de la distribución desigual de la tierra}

El proceso histórico en el ámbito nacional pone en evidencia la configuración de movimientos migratorios desde la colonia puesto que se marcan hitos en los procesos migratorios de acuerdo a grandes acontecimientos que ocurrieron en el país. Sin duda el proceso post colonial, con la masiva venta de tierras públicas sienta las bases para la desigual tenencia de tierras. Se establece la propiedad privada a manos de extranjeros y se crea una ley que impedía a campesinas y campesinos el acceso a la tierra. Además de un desplazamiento espacial de la población campesina que se correspondía a causas económicas como también a cierto temor político (Palau, 2011).

La post guerra significó la promoción estatal de condiciones para la fundación de colonias extranjeras y por ende del latifundio. Fue el Estado quien reconfiguro el régimen de tenencias de tierras (Oddone, 2011) ya que hizo todo lo posible para insertar a migrantes europeos. Esto estaba fundamentado como una necesidad económica paralainserciónyestructuración de una división social del trabajo (Palau, 2011). Los extranjeros migrantes podían tener todas las ventajas, sobre todo en la tenencia de la tierra ya que en el año 1855 se instala la actual Villa Hayes, y se establece como la primera colonia agrícola de extranjeros (Oddone, 2011). Mientras había un aumento de la migración de extranjeros al país, se daba la expulsión (Palau, 2011) de miles de campesinos desposeídos debido a la venta y el cercado de las tierras y cuyo único medio de vida era la agricultura. Dentro de este marco Coronel (1994) señala que en la década del sesenta el país poseía una estructura económica basada en la economía rural sin coincidencia en el tiempo con las sustituciones de importaciones a nivel regional.

En los procesos migratorios internos 
las mujeres fueron afectadas con una invisibilidad social y económica (Riquelme \& Vera, 2015), ya que estas están ubicadas dentro de los roles de cuidado en sus relaciones familiares y comunitarias. La oleada migratoria interna en los años 70 se establece como consecuencia del avance del modelo agroexportador y se intensifica a mediados de dicha década, coincidentemente con la llamada modernización agraria de producción de empresas agroindustriales, en los departamentos de Alto Paraná, Canindeyú, y Amambay.

Finalmente, el acaparamiento de tierras en manos de representantes del modelo agroexportador mediante el avance del modelo productivo es sin duda una de las principales causas del desplazamiento masivo de personas del campo a la ciudad. El asentamiento en las zonas de periferia se fue dando principalmente por el lugar disponible.

Es importante señalar que entre una comunidad yotra no hay marcas territoriales muy visibles geográficamente y que puedan ser delimitadas claramente puesto que la comunidad no necesariamente debe estar conformada siempre a nivel de espacio geográfico, sin embargo, cada zona tiene su historia, tiene características distintivas que las pobladoras y los pobladores marcan en cualquier comunicación con las demás personas fuera de sus barrios. (Muriel del Cueto, s.f ).

\subsection{Asunción, la histórica madre de la desigualdad}

La ciudad de Asunción se compone por 68 barrios, de los cuales solo 3 comunidades del Bañado Sur están señaladas en los mapas oficiales, las demás comunidades se encuentran invisibilizadas. Los bañados sufren la constante problemática de las inundaciones debido a la creciente del río Paraguay, al igual que otras ciudades del país, pero los bañados siempre han sido los más señalados por la sociedad en general por estar ubicados en tierras municipales. Sin embargo, cabe señalar que los antecedentes de referencia son escasos y se expresa la necesidad de que cada territorio cuente con antecedentes históricos debidamente sistematizados, más allá de la oralidad.

Los bañados de Asunción son sitios considerados muy intervenidos por instituciones no gubernamentales (Ongs) y con escasas intenciones focalizadas de parte del Estado con sus órganos centrales y descentralizados, contienen similitudes con la población campesina; puesto que la construcción territorial se configuró con identidades colectivas comunitaria de migrantes de las zonas rurales.

Los bañados están demarcados en las riberas de la ciudad de Asunción y están denominados como las zonas Norte, Sur, Tacumbú y Chacarita, rodeando así a la capital de la República.

Las primeras ocupaciones en los bañados son espontaneas y se dan en los años 6o, estas ocupaciones están ligadas al problema de la tierra pues son familias campesinas quienes se asientan (Coronel, 1994).

El Equipo Arquidiocesano de Pastoral Social, EAPS (1986) menciona un factor importante en cuanto a la permanencia de pobladoras y pobladores en los bañados. El factor con mayor centralidad es el arraigo, en términos específicos es la proximidad con las vecinas y los vecinos, que a su vez en muchos casos son parientes.

En cuanto a las viviendas EAPS (1986) si bien son precarias debe tenerse en cuenta que las inundaciones afectan de sobremanera cualquier mejora que quiera o se haya hecho en las casas en cualquier momento de la historia de los Bañados, es por esto que el conflicto por la propiedad de la tierra fue y sigue siendo una constante.

En 1979 muchas familias de la comunidad Yukyty, localizada al pie del cerro Lambaré en ese entonces, fueron presionadas para salir de sus casas. Desde ese entonces existía una fuerte presión para la inserción de capital privado con intereses inmobiliarios. En 1983 (EAPS, 1986) la municipalidad de Asunción 
prohíbe que vuelvan a sus terrenos. Los pobladores y pobladoras se resisten hasta que los tractores de una empresa derriban sus casas. Las 70 familias existentes fueron reubicadas en la ciudad de Villa Elisa.

La comunidad realiza un proceso de reconstrucción desde adentro (EAPS, 1986) donde se generan necesidades y mediante la autogestión logran costear los servicios e infraestructuras en la medida del crecimiento poblacional de la comunidad reasentada.

En este reasentamiento $y$ relocalización se da un proceso en el que las personas son asignadas a espacios en los cuales vivir y con quienes vivir, es decir no tuvieron participación.

En cuanto al proceso de organización de las pobladoras y los pobladores, se sitúan entre los primeros antecedentes de las comisiones de fomento. Estas tenían estrecha relación con el partido colorado (Coronel, 1994). En ese momentotambién se señala el fenómeno de clientelismo político existente en las zonas, las seccionales pertenecientes al partido colorado tenían un mayor manejo además de territorio; es decir, una dominación (EAPS, 1986).

En el año 1983 la inundación (Coronel, 1994) afectó a 100.000 personas $y$ es en este contexto donde surgen las dos organizaciones referentes de la mano grupos eclesiales y Organizaciones no gubernamentales. La COPZI (Coordinadora de poblaciones de zonas inundables) y la COFASIVI (Coordinadora de Familias sin vivienda) estaban en sintonía con el movimiento barrial latinoamericano $y$ fueron espacios fundamentalmente de organización, las pobladoras y los pobladores organizando incluso movilizaciones en los años 1989 y 1990.

Mediante el quiebre de la hegemonía colorada y del bipartidismo post dictadura (Coronel, 1994) con la mano del movimiento Asunción Para todos en la administración pública, el gobierno de Filizzola Pallarés fue quien acompañó el proceso de organización popular de los bañados y fue la única administración que hasta ese entonces había otorgado participación al sector popular, es decir los bañados con sus comisiones vecinales y grupos de vecinas en igualdad de condiciones participaban frente a otros barrios en los procesos de construcción de políticas públicas.

En el periodo queabarca de 1991 a 1996 la defensa costera fue un punto importante de articulación de comisiones vecinales y grupos de los barrios, ya allí se habían planteado que, si bien era una zona urbana, allí se desarrollaba la lucha por la tierra y por la vivienda digna. Finalmente, los problemas inmediatos como permanentes para el grupo organizado de vecinos lograron ser una fuerza movilizadora.

\section{Objetivos}

Describir las características sociodemográficas de mujeres migrantes del campo a la ciudad que residen en el Bañado Sur.

Caracterizar la configuración del proceso de migración interna de mujeres del Bañado Sur de Asunción.

\section{Metodología}

Estudio cualitativo descriptivo observacional de corte transversal. El enfoque cualitativo como un proceso interpretativo donde se observa el mundo empírico vinculado al estudio de categorías analíticas. Este mundo construido desde puntos de vistas y perspectivas (Hernández et al., 2006). La información de un estudio transversal se recolecta en el presente y, en ocasiones, a partir de características pasadas o de conductas o experiencias de los individuos (Sánchez Hernández, 2014). En este estudio se realizó un muestreo intencional en base a criterios de inclusión. Las personas parte del estudio han sido mujeres residentes en Bañado Sur de Asunción cuyas experiencias se recolecto mediante 12 entrevistas semiestructuradas a profundidad con una guía de preguntas abiertas dirigidas, las entrevistas se realizaron en domicilios particulares de acuerdo a la comunidad de actual pertenencia dentro del Bañado Sur. Previa 
concreción y firma del consentimiento informado las entrevistas fueron grabadas, posteriormente se realizó la desgrabación y la transcripción textual de las mismas. los datos recolectados fueron volcados en matrices por categorías para su análisis descriptivo. En el proceso se respetó la confidencialidad de los datos, la protección de la identidad de las informantes puesto que las matrices fueron configuradas con códigos según cantidad de entrevistas realizadas. En la presentación de los datos se procuró estar en el marco de parámetros del uso de lenguaje no sexista.

\section{Resultados}

Para la descripción de las características sociodemográficas se tuvieron en cuenta las dos áreas, tanto la rural considerada como la de origen y la urbana considerada como la actual.

\subsection{En lo que tiene que ver al área rural se toma la experiencia de la comunidad de origen}

Puesto que las mujeres bañadenses partes del estudio, en primera instancia, se reconocen como campesinas migrantes a la ciudad.

Los desplazamientos han sido desde ambas regiones del país, es decir desde la región occidental como desde la región oriental. En este estudio las entrevistadas han señalado que han llegado al Bañado Sur desde los departamentos de Caazapá, Cordillera, Concepción, Ñeembucú, Caaguazú y Paraguarí; desde la región occidental del departamento de Alto Paraguay.

En cuanto a las condiciones de las viviendas y su materialidad estas estaban elaboradas con materiales diversos como paja (kapi'i), el karanda'y y eternit en los techos; las paredes constituidas de madera, cemento, adobe o tacuaras. En su mayoría las viviendas tenían una o dos habitaciones para toda la familia, y en cuanto los servicios de saneamiento en su mayoría contaban con letrina fuera de la vivienda, el servicio de energía eléctrica era insuficiente, en algunas viviendas se utilizaban lámparas a querosén.

La participación dentro del sistema educativo está vinculada a las condiciones de accesibilidad de las familias campesinas y sus hijas e hijos a la escuela. El traslado hasta las instituciones educativas era una dificultad, ya que los tramos de distancia desde las viviendasa las escuelas implicaban recorridos extensos. Llegar a las escuelas solo era posible caminando o a caballo, los accesos; es decir, los caminos por donde transitar no se encontraban en condiciones óptimas, sobre todo en días de lluvias.

Las entrevistadas comentaron que poseen instrucción escolar primaria, y algunas lograron concluir el bachiller, sin embargo, en su mayoría atravesaron una deserción escolar en la educación escolar básica en el área rural.

En cuanto a la composición familiar en su mayoría se corresponden a familias nucleares extensas, las familias estaban compuestas por madres, padres con una cantidad de entre 5 hasta 15 hijos, todas las familias se dedicaban a la agricultura familia campesina.

Si bien las mujeres cumplían roles asignados socialmente- como el del cuidado en los hogares, también realizaban trabajos de finca para la producción agrícola. Las niñas y niños eran participes del trabajo en ciertas ocupaciones tanto en la casa como en la chacra.

Existía una distribución de las tareas domésticas en el trabajo: el cultivo de alimentos era exclusivo para la alimentación y la subsistencia familiar pero también se reconoce que la producción era para el intercambio con vecinas/os y o parientes. Sin embargo, también se realizaban otros trabajos que les garantizaba el dinero; en sus comunidades existía una distribución de ocupaciones posibles a ser ocupadas para generar dinero, la pesca o el trabajo de capataz estaban asignados a hombres, las mujeres trabajaban en el área de servicios, específicamente en el trabajo doméstico o como pequeñas comerciantes.

En cuanto a la tenencia de la tierra 
respecto a la propiedad y titulación, las familias poseían de entre 4 a 20 hectáreas para el desarrollo de su vida diaria, solo algunas tenían título de propiedad, parte de las tierras de estas familias eran destinadas el cultivo como también para el establecimiento de sus hogares. El acceso a estas tierras fue diverso, ya que registran la compra, herencia, arrendamiento o préstamo.

Sobre las condiciones de atención a la salud en su mayoría destacaron que la dificultad más grande se daba en torno al acceso y a los largos tramos a transitar para llegar a los establecimientos de salud. El medio de transporte era variado según las zonas geográficas, patrulleras, canoas, autos y carretas eran los medios de movilización de las enfermas y los enfermos.

Si bien existían transportes de larga distancia, estos tenían horarios fijos, lo que era un condicionante para la movilidad de quienes estaban enfermas/os o debían de tener partos en el caso de las mujeres. Se menciona eran trasladadas fuera de la comunidad si el estado de salud era más grave, pues en casos de menor gravedad se recurría con personas de la misma comunidad quienes tenían conocimientos del uso de medicamentos químicos como de hierbas medicinales que crecían en sus propias fincas.

Se identifican espacios comunitarios que son a su vez espacios de encuentro establecidos en una zona geográfica específica, las fiestas patronales, las actividades deportivas como un partido de futbol, reuniones políticas, y en comités de productores/as fueron reconocidos.

Establecerse en la periferia de la ciudad de Asunción fue una necesidad de asentamiento post proceso de migración, hecho que hizo posible la conformación de las comunidades en el Bañado Sur.

En esta investigación las participantes pertenecen a las comunidades de Yukyty, San Miguel, Porvenir, San Blas y San Cayetano.

En lo que tiene que ver con las condiciones de habitabilidad $y$ accesibilidad, las viviendas en el Bañado Sur fueron construidas durante el proceso de asentamiento en simultáneo con la constitución de las comunidades.

Las viviendas, específicamente, fueron construidas por partes; con materiales mixtos para realizar las diversas estructuras, los materiales como hule (polietileno), terciada, madera, ladrillos, cemento y en algunos casos las viviendas se construyeron con ladrillos. Muchos de esos materiales eran reciclados del vertedero Cateura.

Para la construcción de las viviendas se hizo necesario lograr la estabilidad del suelo. Para este proceso se cargaron camionadas de escombros, las entrevistadas mencionaron que mínimamente se debían cargar 35 a 45 cargas por cada vivienda para lograr la estabilidad de la tierra, puesto que en ese entonces era muy inestable y ante cada inundación era una necesidad para las familias mantener el suelo.

Las mujeres con niñas/os movilizaban los escombros en baldes, los días de lluvia representaban una gran dificultad ya que quienes vivían cerca de los arroyos se encontraban con los desbordes que inundaban sus viviendas constantemente.

Se reconoce que actualmente hay más facilidades para que los camiones logren entrar hasta cada vivienda inclusive en las comunidades más alejadas.

Sin embargo, esto antes no era posible, ya que no había accesos es decir no había calles o estas eran muy estrechas haciendo imposible el tránsito de camiones de gran tamaño, por lo que las calles también fueron una construcción de las pobladoras y los pobladores.

En su mayoría las viviendas fueron mejorando de acuerdo al crecimiento de la comunidad, incorporando materiales de concreto a sus estructuras. Aun así, persiste el deterioro de las estructuras por las inundaciones, haciendo que las familias tengan dudas del retorno a sus viviendas por las condiciones en las que las encuentran y la inversión que implica reestablecerse en su comunidad luego de cada inundación. 
Desde la carga de escombros para la estabilidad del suelo hasta la movilización de sus pertenencias de los refugios hasta sus casas son factores que condicionan la calidad de vida.

Habitar el Bañado Sur entonces se trata de una supervivencia entre la autogestión y la solidaridad, mencionan además las entrevistadas que, al vender sus terrenos en zonas rurales, debieron asentarse en las ciudades en espacios mucho más pequeños y eso lo tienen en cuenta.

El agua potable no era accesible para todos y todas en el barrio, a pesar de los bañados fueron ocupados precedentemente recién en los años noventa se establecieron las comisiones vecinales que conjuntamente con sacerdotes jesuitas lograron construir un sistema de distribución de agua a través de manzaneros/as voluntarias/os que autogestionadamente y con aportes de las vecinas y los vecinos lograron comprar tubos y establecer un sistema de distribución del agua.

Con antecedentes de dificultades de permanencia en el sistema educativo formal en el área rural, post-migraciones estas mujeres se registran en una reinserción escolar con extraedad tanto en la secundaria como estudios terciarios y de oficios que no lograron culminarse.

Otro factor muy importante en la concreción de los estudios primarios como secundarios de las mujeres es la empleabilidad ya que existe una necesidad del ingreso personal y familiar, lo que hace que el contar con un trabajo sea una prioridad.

Las familias son extensas y monoparentales, en su mayoría son mujeres quienes están a cargo de sus hijas/ os e incluso de adultos mayores, pero resulta que existe una diversidad en cuanto a la constitución familiar, sin embargo es predominante la jefatura de hogar por parte de las mujeres, manifestaron tener de entre 3 a 17 hijos pero que en la actualidad son de 2 a 7 hijos/as, mayoritariamente menores de edad quienes permanecen en las viviendas ya que están insertos/as en procesos escolares en las comunidades.

El desempeño en el área laboral actual de las mujeres bañadenses, se ubica en su mayoría en las tareas domésticas no remuneradas, realizan el trabajo de cuidado a niños/as, adultos mayores, y a sus propias parejas, además de retomar las tradicionales tareas del campo con la cría de animales para la posterior comercialización de productos y el autoconsumo.

Realizan además trabajos independientes en sus hogares como la costura, haciendo confecciones que les permiten tener ingresos; además de todo esto, se desempeñan como referentes de la comunidad, agentes de servicios estatales, pero sufriendo la precarización del trabajo, sin seguro social y otros beneficios sociales.

Cabe destacar que la mayor fuente de generación de ingresos sigue siendo el reciclado de residuos sólidos, de forma independiente o sujeta a condiciones del vertedero Municipal Cateura. Este trabajo desarrollado en circunstancias sumamente precarias, sin seguro social y remuneración digna. Todas las áreas laborales que se describen anteriormente se enmarcan dentro del trabajo informal.

En cuanto a la tenencia de la tierra se indica que, si bien son tierras municipales sin titulaciones, las mismas en algunos casos han sido adquiridas por herencia; o por medio de compra a dueños/as anteriores que ya estaban asentados en el barrio. Establecerse en los bañados corresponde una necesidad de encontrar un espacio accesible donde vivir posterior a la migración de las comunidades de origen.

\subsection{Aproximaciones al proceso de migración interna al Bañado Sur}

Se presenta un esquema elaborado que menciona a 6 (seis) momentos identificados como partes del proceso de migración interna que configuro el tránsito de las mujeres hasta la ciudad (Figura 1).

En torno a estos 6 (seis) momentos identificados como partes del proceso de migración interna son varias causas las que se describen como motivos de migración 
Figura 1. Proceso migratorio interno. Etapas del proceso de migración interna / rural urbana.

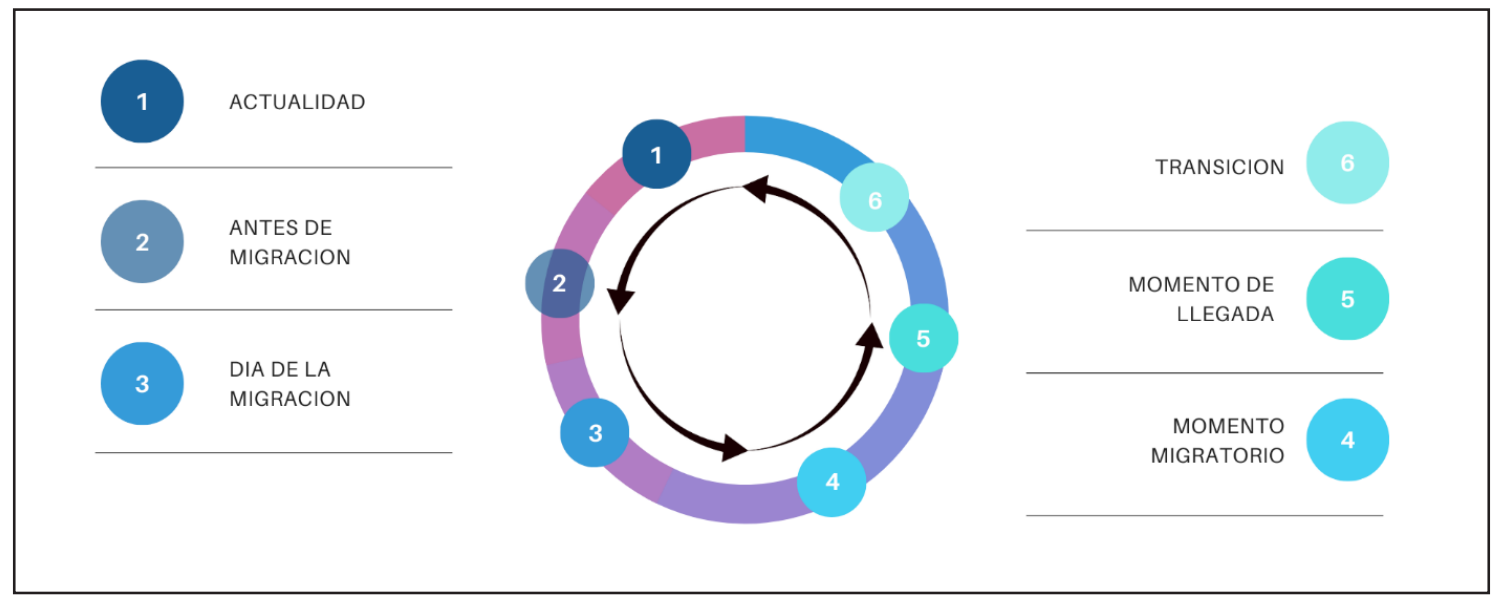

entre las cuales se mencionó principalmente la búsqueda de espacios y oportunidades laborales, ya que estas mujeres realizaron las movilidades interurbanas e incluso en otros departamentos antes de reasentar en el Bañado Sur sin embargo no encontraron puestos laborales.

Así es que se optó entonces por la migración hacia la capital es decir Asunción, se hace énfasis que la obtención de una actividad laboral remunerada responde a la necesidad de mejorar las condiciones de vida de la familia y que en la capital podrían encontrarse estas oportunidades.

"Vine a trabajar y me tuve que quedar" es lo manifiestan en su mayoría las mujeres, se insertaron en el trabajo informal, donde encontraron múltiples dificultades, desde el desconocimiento del funcionamiento y la dinámica de una ciudad, hasta encontrarse solas sin ningún familiar en el momento de llegada. En la búsqueda de una independencia económica con o sin sus parejas realizaron el proceso de movilidad hacia la capital.

Mencionaron que, en el caso de haber sido niñas cuando migraron, fue una decisión establecida en general por la familia, y en específico de las madres. Fueron ellas quienes les comentaron de tal determinación, pero de más adultas habían comprendido que la migración se dio por problemas de la tierra en el campo, es decir por la propiedad ya sea por la tenencia, por herencia y permanencia en las comunidades; por el uso de la tierra es decir por las actividades agrícolas que no se consideraban como rentables, también se mencionan motivos de persecución política.

El traslado desde la comunidad de origen hasta el momento de partida se dio en la mayoría de las veces en compañía, es decir alguna persona las escolto hasta el punto de partida y las despidió - en algunos casos nunca más pudieron volver- es decir, desde sus hogares hasta el lugar donde tomaron el trasporte que las movilizo hasta la ciudad.

Las viviendas se encontraban lejos de denominada popularmente como "parada" o salida de los buses, los caminos se encontraban en mal estado ya que eran de arena roja, en los días de lluvia, eran intransitables.

La terminal de ómnibus de Asunción es la última parada que todas mencionan en su arribo hacia la ciudad. El medio de transporte utilizado más citado fue el ómnibus de larga distancia, estos buses tenían horarios específicos de salida.

El traslado hasta Asunción tuvo una duración con aproximaciones de entre 7 horas y en adelante llegándose a mencionar 
hasta 12 horas, también llegar a la capital implico días en el caso de quienes vinieron en barco.

Desde la comunidad de origen se dieron una o dos movilidades antes de la inserción y reasentamiento definitivo en el bañado Sur.

En primera instancia el lugar de llegada se estableció en el departamento central y en algunos casos en la capital, el bañado Su fue el último lugar de llegada y el inicio de una vida.

La inserción laboral en el proceso de instalación en la ciudad para las mujeres estuvo marcada por experiencias nuevas. El ser joven y con baja instrucción escolar solo podrían ubicarse en el trabajo de cuidado, ya que era la única oferta que encontraron, además de que el idioma fue también un determinante ya que el guaraní es el idioma materno. Empleadas domésticas sin retiro, niñeras, cocineras, se dedicaron al arte, a las ventas en los mercados 4 y de abasto ubicados en Asunción, realizaron un sinfín de actividades laborales.

Un dato resaltante es que quienes vinieron de niñas o adolescentes al Bañado comentan que esa etapa estuvo marcada por el trabajo casi obligatorio, - como el de todos los miembros de su familia- ya que sus trabajos contribuían a la sobrevivencia de su propia familia que ya no contaba con la tierra para labrar y generar alimentos, así las familias recurrieron a la venta de menudencias en las calles, a la venta de frutas, venta de agua "fresca" en cantaros" ; e incluso se mencionan casos de criadazgo.

Ya en el Bañado, el reciclado de residuos fue una opción casi inmediata. Incluso con hijos/as las mujeres decidían salir a recoger los materiales reciclables por las calles de Asunción hasta llevarlos en el vertedero municipal Cateura para su comercialización.

Con relación a qué expectativas tenían al venir a la ciudad, es decir cómo se sentían anímicamente, manifestaron que sintieron de todo; desde felicidad, porque tenían una idea diferente de lo que podría ser la ciudad; frustración, porque al realizar el desembarque en la última parada en la terminal de ómnibus no era la ciudad un lugar similar según la idea que tenían en mente en sus comunidades rurales.

En la transición de establecerse sintieron miedo, el miedo que representaba estar situadas en un lugar por completo desconocido, sin poder saber el funcionamiento de todo el funcionamiento de la ciudad, sobre sus códigos y sin un espacio de referencia al cual recurrir para contar con información y en lo particular desde el uso del leguaje hasta el uso transporte público.

Consideraron que la experiencia fue muy dura tanto para ellas como para sus madres, quienes permanecieron en la comunidad campesina sin comunicación. Las madres observaron cómo sus hijas se trasladaban hacia otra vida en medio de la incertidumbre.

Sobre cómo pensaban a la ciudad, entre risas comentaron que hacían comparaciones de sus comunidades e "imaginaban a la ciudad de una forma distinta", con departamentos altos, sin tantos arbustos y árboles como en sus comunidades.

Reconocen que se crearon percepciones a través de otras personas que establecieron en su imaginario lo que es y lo que sería el ir a vivir en la ciudad; más aún en la idea de lo que se trataba la capital de un país. Algunas mujeres no tenían idea de que se trataba ir a Asunción y sus implicancias y mencionan que era un espacio mucho más pequeño de lo que consideraban.

De jóvenes y de adultas recibieron un trato discriminatorio por decidir vivir en el Bañado Sur, inclusive por parte de sus familiares quienes habitan fuera de él. Refieren que tuvieron una percepción equivocada sobre cómo eran las comunidades del Bañado debido a la estigmatización que se genera en torno a la zona y sus habitantes.

Todos estos hechos las marcaron profundamente ya que, en el Bañado, además de un espacio para vivir fue un lugar donde encontraron todo tipo de 
ocupaciones para poder sobrevivir día a día con su propio trabajo.

Teniendo en cuenta que cada espacio fue construido con este mismo esfuerzo, las viviendas fueron hechas sobre múltiples cargas de escombros en lo que eran humedales/ lagunas, lo que supone un gran esfuerzo propio y colectivo.

En cuanto a la participación en actividades comunitarias, mencionan que las mujeres son las principales en la asunción de todo tipo de roles en las distintas actividades del barrio. Son las que más opinan y proponen, porque conocen las realidades del día a día.

Comisión de padres en instituciones educativas, comisiones vecinales, participación eclesial, servicio comunitario, dirección de organización barrial, comité de mujeres, grupo de emprendedoras, trabajadoras de la salud, recicladoras organizadas, grupo deportivo y cooperativa de vivienda son los espacios organizativos que mencionaron las entrevistadas. Espacios de los que forman parte además de realizar las tareas de cuidado, trabajo doméstico y trabajo remunerado suponiendo una triple jornada de trabajos.

La dependencia económica de sus parejas existe, pero sus ingresos no son suficientes; por lo cual realizan diversas actividades laborales para generar una mayor entrada de dinero de manera a satisfacer necesidades específicas.

Finalmente, cuanto a la organización barrial lo primero que señalan es que existen muchas familias, que el número exacto es siempre desconocido, que no hay datos oficiales. El barrio y las comunidades se constituyeron gracias al trabajo de vecinos y vecinas que, en comisiones de agua, camino $y$ vecinales en general construyeron todo lo que hoy se ve en el Bañado Sur.

Las entrevistadas recalcan que existe una latente amenaza de desalojo para llevar a cabo proyectos inmobiliarios. En el caso de que tales proyectos se realizasen generaría un doble despojo, teniendo en cuenta que ya migraron del campo.

Es posible que vuelvan a migrar a otros espacios, y que el único camino para frenar esto, consideran, es la organización, la unidad bañadense ante la constante amenaza.

La organización territorial hace lo que está en las medidas de sus posibilidades, y con la gente que hace parte de ella, cumple la tarea de informary establecer discusiones en las comunidades. Aquí, las mujeres son de fundamentales, ya que ellas son las que participan mayoritariamente y además encabezan las organizaciones.

La defensa costera, la titulación de las tierras, la recuperación de espacios públicos, el respeto al arraigo y la organización que construyó el barrio son demandas que mencionan las mujeres bañadenses y que no hay disposición para salir del lugar ya que este fue construido con el propio esfuerzo, con solidaridad y trabajo colectivo comunitario.

Ante estas situaciones difíciles como también en duelos o enfermedades la ayuda mutua es una reacción casi natural de vecinos. No pocas veces se realizan actividades de recaudación para las familias en dificultad. Ante los innumerables problemas notoriamente la solidaridad es un valor y práctica constante.

También se observa que en ocasiones llegan nuevos/as habitantes del Bañado Sur desde el interior del país. Estas personas son orientadas por los mismos vecinos y vecinas sobre el cómo establecerse en la ciudad y en el barrio. Aquellos manifestaron que en su llegada al barrio recibieron buen trato e incluso la misma gente de la comunidad les ayudo a tener sus primeras pertenencias. Fueron los habitantes del Bañado quienes les tendieron una mano en momentos difíciles, desde la colocación de cables de energía eléctrica hasta el agua de pozo que compartían con todo el barrio.

Las mujeres estuvieron frente a las movilizaciones, marchas y acciones en torno a una respuesta mínima del Estado. Ellas son quienes movilizan a sus familias en las inundaciones, organizan sus comunidades y coordinan las actividades.

Las inundaciones y todo lo que ellas 
conllevan afectan a las familias de gran manera, desde los ingresos económicos hasta sus rutinas más básicas y hogareñas, sometiéndolas a una situación de stress que hace que algunas familias decidan volver a sus comunidades de origen, o desplazarse a cualquier otro lugar disponible sin retornar al barrio vendiendo todas sus cosas volviendo a migrar a otra parte del país, o de la capital.

Las familias han construido cada barrio, pero las mujeres fueron claves para la existencia de espacios como las escuelas, capillas, guarderías y plazas. El bañado Sur es símbolo del trabajo de quienes lo habitan, las mujeres, las sociedades no están completas y no pueden progresar. Así, el Bañado como comunidad, no se sostiene si no están ellas; quienes con sus hijos e hijas levantaron sus casas, las calles y las comunidades.

\section{Conclusiones}

La dictadura también reafirmó las bases del latifundio con los conocidos programas de colonización, además se dan cambios productivos e insertan a la población campesina dentro de la dinámica del mercado capitalista en simultaneo con la inserción agroexportadora al campo, que inicia el ciclo agrícola con cultivo de soja y se da su rápida expansión y genera grandes impactos sociales.

Se generó un proceso de expulsión campesina y migración hacia las ciudades, creando un reasentamiento en las zonas inundables de la ciudad de Asunción hoy denominadas Bañados.

Respecto a las características sociodemográficasdelasmujeresseobservan concomitantemente desigualdades sociales en las realidades urbanas y rurales que habitan y habitaron las mujeres. Según la literatura existe una feminización de los procesos migratorios que son expresiones del funcionamiento desigual del modelo de producción; sin embargo, se debe recalcar que estas migraciones internas no tuvieron consecuencias similares para hombres como para las mujeres, ya que estas poseían condicionantes estructurales que incidieron en su reasentamiento en el Bañado Sur.

La migración interna como experiencia de las mujeres se relaciona directamente con desigualdad estructural que opera en la sociedad paraguaya, los asentamientos periféricos, que a su vez son productos del desplazamiento forzado causado por la expulsión de las poblaciones campesinas, y que en Paraguay la tierra es además de ser medio de vida, es relación social que se quiebra cuando se dan estos desplazamientos forzados, producidos por el modelo agroexportador.

En este sentido las migraciones no se dieron directamente desde las comunidades de origen hasta el bañado sur, sino que en un primer momento se dieron procesos de migración de comunidades campesinas hacia centros urbanos distritales $y$ departamentales como hacia la capital.

La autopercepción que expresan las mujeres bañadense del sur condice a una identidad de herencia campesina, mediante la organización en el territorio contribuyó también a una re conceptualización de lo que significo migrar y construir el barrio donde habitan las mujeres y sus familias.

De acuerdo al contexto nacional y la distribución desigual de la tierra en Paraguay, hace que hoy los bañados estén en conflicto por el derecho a vivir en la ciudad. Constantemente las comunidades son amenazadas a ser parte de una nueva expulsión, las mujeres bañadenses son quienes se encuentran enfrentadas con las corporaciones inmobiliarias y proyectos por parte del Estado, las mismas manifiestan que las instituciones estatales que no dan respuestas ante las múltiples necesidades y que esto es una constante histórica y no reciente pese a que el proceso de migración hacia el bañado sur no ha permanecido estático, el estado con sus instituciones no han logrado establecer intervenciones integrales con esta importante población de la capital del Paraguay. 


\section{Referencia}

Antonelli, R. A. (2016). La periferia de la periferia: mujeres migrantes en el conurbano bonaerense. http:// conferencias.unc.edu.ar/index.php/ gyc/4gys/paper/viewFile/4397/1476

Carpinetti, J. (2017). Teorías de las migraciones, paradigmas epistemológicos en las ciencias sociales y condiciones sociales de producción. Cuadernos FHyCS-UNJu - Universidad Nacional de Jujuy, (52), 59-79.

Coronel, B. (1994). El movimiento Barrial en Paraguay Un nuevo actor social. Documento de trabajo $N^{\circ} 65$ Programa de Estado y Sociedad. Base Investigaciones Sociales.

Equipo Arquidiocesano de Pastoral Social. (1986). Lucha y tierra urbana en Asunción. El derecho de los pobres a la tierra y la vivienda, Araverá.

Falú, A. (2018). Espacios metropolitanos igualitarios. Barcelona: asociación mundial de las grandes metrópolis.

Gómez Walteros, J. A. (2010). La migracion Internacional: Teorias y enfoques, una mirada actual. http://www.redalyc.org/ articulo.oa?id=165014341004, 13(26), 81-99.

Henault, M. (1973). La mujer y los cambios sociales. En H. M. Morton, Las Mujeres dicen Basta (p. 13-40). Ediciones Nueva Mujer.

Hernández, R., Fernández, C., \& Baptista, P. (2006). Metodología de la investigación (4 $4^{\mathrm{a}}$ ed.). Mc Graw Hill.

Marini, R. M. (1993). El desarrollo del capitalismo mundial y su impacto en América Latina. El desarrollo del capitalismo mundial y su impacto en América Latina Archivo de Ruy Mauro Marini. www.archivochile.com/Ideas_ Autores/maurinirm/ozal/maurini_ aloooo2.pdf

Muriel del Cueto, C. (s.f.). Un estudio sobre la acción cultural en dos barrios del Gran Buenos Aires.

Ocampo, M.S.(2015).Del campoalaperiferia de la ciudad, la omnipresente sombra de la violencia. Campesinos desplazados forzados en Colombia caminan de la mano del eterno retorno a la violencia. vulneracion y potencia de vida. http:// www.scielo.br/scielo.php?pid=So103$65642015000200161 \&$ script $=$ sci_ abstract\&tlng=es. 83

Oddone, H. (2011). Impactos de la migración en el desarrollo nacional: una aproximación histórico-social. Migrantes Perspectivas (criticas) en torno a los procesos migratorios del Paraguay (p. 6o-82). Ápé Paraguay.

Osorio, F. (1993). La violencia del silencio. Desplazados del campo a la ciudad. Consultoría para los Derechos Humanos y el Desplazamiento, CODHES. Pontificia Universidad Javeriana, Facultad de Ciencias Económicas y Administrativas.

Palau, T. (2011). El marco expulsivo de la migración paraguaya. Migrantes Perspectivas (criticas) en torno a los procesos migratorios del Paraguay (p. 40-59). Ápe Paraguay.

Palau, T., \& Heikel, M. V. (2016). Los campesinos el Estado y las empresas en la frontera agrícola (2a ed.). Base Investigaciones Sociales.

QuirogaDiaz,N.(2009).Economíafeminista y economía social. Contribuciones a una critica de las nuevas políticas de combate a la pobreza. Iconos. Revista de Ciencias Sociales, 77-89.

Riquelme, Q., \& Vera, E. (2015). Agricultura campesina, agronegocio y migración. CDE.

Salazar Cruz, C. (2000). La relación población-recursos en la periferia urbana. Una experiencia teóricometodológica. Estudios Demográficos y Urbanos. www.redalyc.org/articulo. oa?id=31204505, 641-64.

Sánchez Hernández, V. (2014). Metodología de la investigación, bioestadística y bioinformática en ciencias médicas y de la salud. McGraw-Hill Interamericana. 


\section{Sobre la Autora}

\section{Belinda García}

Licenciada en Trabajo Social por la Universidad Nacional de Asunción, investigadora en el área de género, desarrollo rural y periferia urbana, diplomada en Investigación, Transferencia y Desarrollo en la Universidad Pública por el Consejo Latinoamericano de Ciencias Sociales. 